\title{
EFEITOS DO ESTRESSE OSMÓTICO NO DESENVOLVIMENTO INICIAL DE Phaseolus vulgaris $\mathrm{L}$.
}

\author{
José Lucas dos Santos Oliveira* \\ Edevaldo da Silva*
}

\begin{abstract}
RESUM0: A salinização de solos é uma propriedade química que, especialmente no semiárido nordestino, tem sido um dos problemas que mais afeta a sua fertilidade e, consequentemente, a produtividade agrícola da região. Essa pesquisa teve como objetivo avaliar os efeitos do estresse salino no desenvolvimento inicial do feijão (Phaseolus vulgaris L.), espécie cultivável no semiárido nordestino. 0 experimento foi realizado utilizando concentrações experimentais de cloreto de potássio (KCl), a saber: 0,0; 40,0; 80,0; 120,0; 160,0; 200,0 m mol L-1. Para cada concentração em estudo existiu três subamostras de sementes tratadas com respectiva concentração do sal, sendo avaliadas as seguintes variáveis morfofisiológicas: porcentagem de germinação (\%G), índice de velocidade de germinação (IVG), tempo médio de germinação (TMG), crescimento da raiz e da parte aérea (mm), potencial inibitório (PI) e biomassa fresca e seca da raiz e da parte aérea. 0s dados reportaram que o $\mathrm{KCl}$ exerceu toxidade para a germinação de sementes de $P$. vulgaris, mesmo quando disponível na menor concentração do sal em estudo (40 m mol L-1), reduzindo variáveis como a \%G. 0 IVG foi afetado a partir da concentração de $80 \mathrm{~m} \mathrm{~mol}$ $\mathrm{L}^{-1}$. No mesmo período de exposição ao $\mathrm{KCl}$, houve redução do crescimento da parte aérea e da raiz das plântulas $P$. vulgaris, assim como a diminuição da biomassa fresca e seca das plântulas, com efeitos mais tóxicos do sal representados na concentração de $200 \mathrm{~m} \mathrm{~mol} \mathrm{~L}^{-1}$ de $\mathrm{KCl}$. Solos salinos com concentrações de $\mathrm{KCl}$ acima de 40

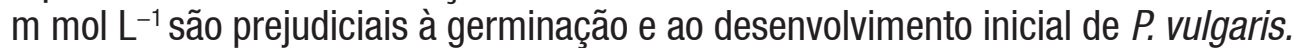

PALAVRAS-CHAVE: Feijão; Germinação; Salinidade; Semiárido.

\section{EFFECTS OF OSMOTIC STRESS IN THE EARLY DEVELOPMENT OF Phaseolus vulgaris L.}

\begin{abstract}
Soil salinity is a chemical factor featuring one of the problems that most affect fertility and agricultural productivity, particularly in the semi-arid region of northeastern Brazil. Current research evaluates the effects of saline stress in the early development of beans (Phaseolus vulgaris L.) sown in the semi-arid region of northeastern Brazil. The experiment comprised experimental concentrations, namely, 0.0; 40.0; 80.0; 120.0; $160.0 ; 200.0 \mathrm{~m} \mathrm{~mol} \mathrm{~L}^{-1}$ of potassium chloride $(\mathrm{KCl})$. Three sub-samples of seeds treated with the respective salt concentration were used for each concentration analyzed. The morpho-physiological variables analyzed were germination percentage $(\% \mathrm{G})$, germination velocity index (GVI), mean germination time (MGT), growth of root and aerial part $(\mathrm{mm})$, inhibitory potential $(\mathrm{IP})$ and fresh and dry biomass of the root and of the aerial section. Results show that $\mathrm{KCl}$ provided toxicity for the germination of $P$. vulgaris seeds even when available in the lowest salt concentration rate analyzed $\left(40 \mathrm{~m} \mathrm{~mol} \mathrm{~L}^{-1}\right)$, reducing variables such as $\% \mathrm{G}$. GVI was affected as from concentration $80 \mathrm{~m} \mathrm{~mol} \mathrm{~L}^{-1}$. Aerial part and of the roots of $P$. vulgaris seedlings decreased at the same period that $\mathrm{KCl}$ was exposed; likewise, fresh and dry biomass of the seedlings, with the salt's more toxic effects in concentration 200 $\mathrm{m} \mathrm{mol} \mathrm{L}{ }^{-1}$ of KCl. Saline soils with $\mathrm{KCl}$ concentrations above $40 \mathrm{~m} \mathrm{~mol} \mathrm{~L}^{-1}$ jeopardize germination and the early development of $P$. vulgaris.
\end{abstract}

KEYWORDS: Beans; Germination; Salinity; Semi-arid region.

\footnotetext{
Doutorado em andamento no Programa de Pós-graduação em Desenvolvimento e Meio Ambiente (PRODEMA) na Universidade Federal da Paraíba (UFPB).

* Doutorado em Química. Docente Adjunto da Universidade Federal de Campina Grande (UFCG) e Docente permanente do Programa de Pós-Graduação em Desenvolvimento e Meio Ambiente (PRODEMA), da Universidade Federal da Paraíba (UFPB), Brasil. Editor-chefe do periódico Acta Brasiliensis. E-mail: edevaldos@yahoo.com.br
} 


\section{INTRODUÇÃO}

As condições edafoclimáticas se constituem como fatores determinantes para a produção das culturas agrícolas, podendo interferir negativamente na produção, causando danos à planta (AQUINO et al., 2017) e, consequentemente, comprometer 0 estabelecimento da cultura no solo.

0 processo de salinização do solo é considerado um dos principais problemas ambientais emergentes que tem causado a infertilidade de solos agrícolas. 0 solo se torna salino quando ocorre a acumulação de sais ao longo do tempo, desencadeando diversos problemas, principalmente nas regiões áridas $\mathrm{e}$ semiáridas (OLIVEIRA et al., 2017).

Na região semiárida do nordeste brasileiro os problemas com salinidade são frequentes, especialmente por que há muitas fontes de águas naturais com elevada salinidade utilizada para irrigação e, essa realidade, tem incentivado a criação de novas tecnologias para minimizar os problemas derivados da salinidade na produção das culturas (SILVA et al., 2018).

As técnicas inadequadas de produção agrícola, como 0 uso intensivo de fertilizantes que possuam elevadas concentrações de sais em sua composição e 0 desenvolvimento impróprio de métodos para a irrigação de culturas, são as principais causas de salinização dos solos (CRUZ et al., 2017).

A concentração elevada de alguns íns como $\mathrm{Ca}^{+2}, \mathrm{Na}^{+}, \mathrm{Mg}^{+2}$ e $\mathrm{Cl}^{-}$no ambiente pode exercer toxicidade às plantas, pois vai favorecer a diminuição de parâmetros essenciais ao desenvolvimento normal dos vegetais, como o potencial hídrico das plantas e osmótico do solo (BEZERRA et al., 2018), além de interferir na fisiologia da planta.

Dentre os processos fisiológicos que são afetados pela concentração salina do solo, está a diminuição da atividade estomática nas plantas, que tem objetivo de reduzir a perda de água, mas, acaba comprometendo processos como a fotossíntese e transpiração do vegetal (OLIVEIRA et al., 2017). Além disso, a salinidade pode interferir na nutrição do vegetal (SOUZA et al., 2018).
Na germinação das sementes, os fatores ambientais são essenciais na determinação da capacidade germinativa. A presença de sais durante a germinação das sementes pode comprometer a absorção de água pela semente e, consequentemente, causar toxicidade (JOSÉ et al., 2016).

Conhecer a tolerância das espécies vegetais à presença de sais é essencial para definir 0 tipo de cultura que deve ser cultivada em determinado ambiente, especialmente em áreas salinas, bem como saber as técnicas de manejo e irrigação adequadas para cada ambiente evitando assim a intensificação da salinidade (PEREIRA et al., 2017).

0 feijão (Phaseolus vulgaris) é uma cultura de grande importância no Brasil. Por isso, os métodos de produção, qualidade do solo e da água são determinantes para a eficiência de produção da cultura, visto que esses fatores afetam diretamente a germinaçãa e o crescimento dessas plântulas (DALCHIAVON; NEVES; HAGA, 2016).

Essa pesquisa teve como objetivo avaliar os efeitos do estresse salino no desenvolvimento inicial do feijão (Phaseolus vulgaris L.), espécie cultivável no semiárido nordestino.

\section{METODOLOGIA}

Para 0 desenvolvimento experimental, foram utilizadas sementes de $P$. vulgaris, adquiridas na Empresa Brasileira de Pesquisa Agropecuária (Embrapa) que corresponderam à variedade BRS Estilo.

Após a aquisição das sementes, foi realizado inicialmente um teste de germinação, seguindo as determinações da Regra de Análises de Sementes (BRASIL, 2009) com objetivo de avaliar a capacidade germinativa das sementes.

As sementes foram desinfetadas em hipoclorito de sódio ( $\mathrm{NaClO}$ ) a 1\% por 10 minutos e, em seguida, lavadas três vezes em água destilada para iniciar o experimento com 0 sal. Para avaliar possível estresse osmótico na variedade de $P$. vulgaris, foi utilizado o cloreto de potássio (KCl), em concentrações 
experimentais, a saber: 0,0; 40,0; 80,0; 120,0; 160,0; $200,0 \mathrm{~m} \mathrm{~mol} \mathrm{~L}^{-1}$.

Em seguida, as sementes de $P$. vulgaris permaneceram em contato por 20 minutos em cada respectiva concentração de $\mathrm{KCl}$ estudada, e colocadas para germinação em placas de Petri, com camada dupla de papel de germinação. Cada placa de Petri recebeu 0 equivalente a $8 \mathrm{~mL}$ de cada concentração de $\mathrm{KCl}$.

0 experimento foi desenvolvido em triplicatas, onde cada placa conteve 25 sementes e após serem colocadas para germinação a placa foi isolada com plástico filme, permanecendo em temperatura ambiente (média de $30^{\circ} \mathrm{C}$ ) durante 0 decorrer de todo 0 experimento (144h).

Foram analisadas diferentes respostas fisiológicas da germinação e crescimento inicial que pudessem indicar toxicidade do $\mathrm{KCl}$ em $P$. vulgaris: porcentagem de germinação (\%G) (BRASIL, 2009), crescimento da raiz e da parte aérea $(\mathrm{mm})$ quantificado com 0 uso de um paquímetro digital, índice de velocidade de germinação (IVG) (MAGUIRE, 1962), tempo médio de germinação (TMG) e potencial inibitório (PI) do sal na germinação das sementes. Além disso, foi avaliada também a biomassa fresca e seca das plântulas ao final do experimento.

Os parâmetros fisiológicos acima descritos foram analisados todos os dias durante todo o período que compreendeu 0 experimento. Sendo realizada apenas uma biometria após 144h de exposição ao KCl, que correspondeu ao último dia do experimento.

As análises estatísticas foram realizadas no software SPSS 20.0, por meio da análise de regressão polinomial para avaliar possível correlação entre 0 aumento da concentração de $\mathrm{KCl}$ e diminuição/ aumento das variáveis fisiológicas analisadas. $A$ análise de variância entre as médias foi por meio da ANOVA, seguida do teste de Tukey, considerando nível de significância $p<0,05$.

\section{RESULTADOS}

As sementes de $P$. vulgaris expostas a concentrações experimentais acima de $80 \mathrm{~m} \mathrm{~mol} \mathrm{~L}^{-1} \mathrm{de}$ KCl tiveram redução significativa do IVG após $144 \mathrm{~h}$ de exposição ao sal (Figura 1). Para o mesmo período de tempo, o TMG foi mais elevado na concentração de $200 \mathrm{~m} \mathrm{~mol} \mathrm{~L}^{-1}$ de KCl, dobrando 0 seu valor em relação ao observado no controle (Figura 2).

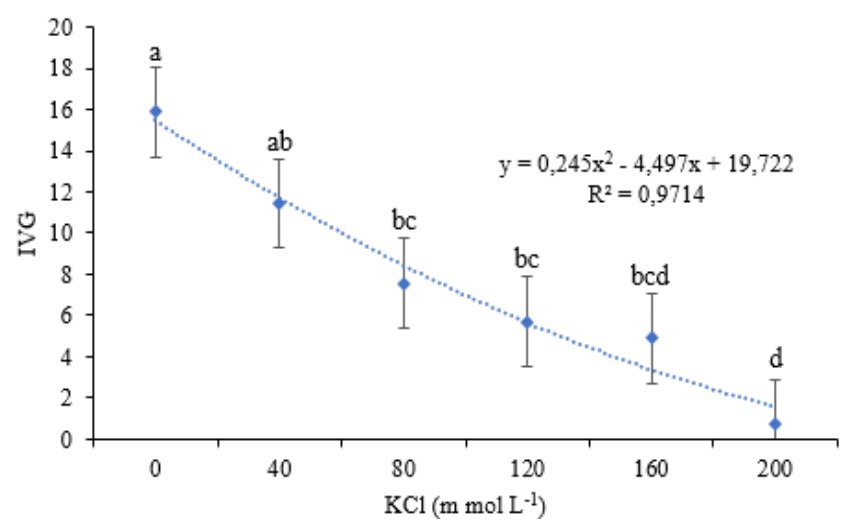

Figura 1. IVG de sementes de $P$. vulgaris expostas (144h) a concentrações experimentais de KCl. Letras minúsculas iguais, para a mesma coluna, indica que os valores médios não apresentaram diferenças estatísticas pelo teste de Tukey $(\mathbf{p}<0,05)$.

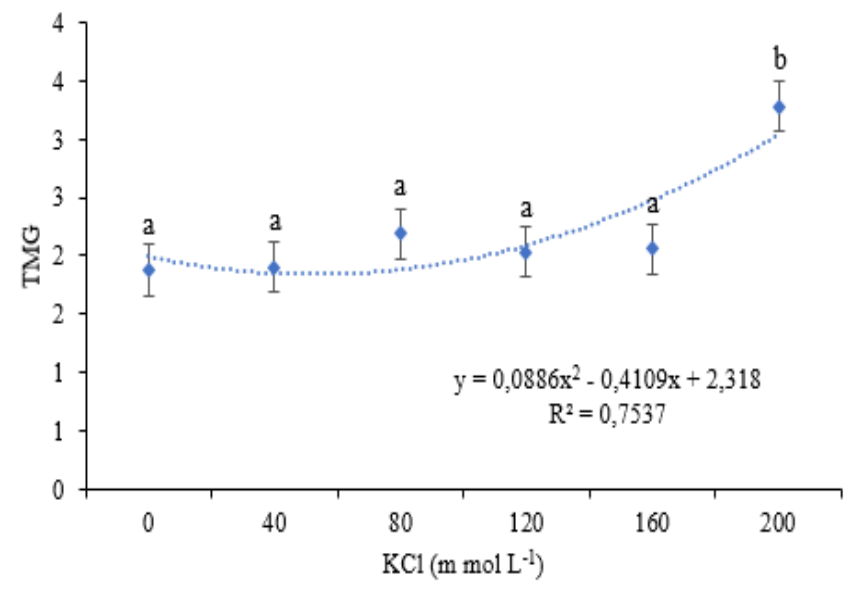

Figura 2. TMG de sementes de $P$. vulgaris expostas (144h) a concentrações experimentais de $\mathrm{KCl}$ Letras minúsculas iguais, para a mesma coluna, indica que os valores médios não apresentaram diferenças estatísticas pelo teste de Tukey $(p<0,05)$. 
Houve correlação entre a redução do crescimento da raiz e da parte aérea nas plântulas de $P$. vulgaris, e 0 aumento das concentrações de $\mathrm{KCl}$, observados após 144h de exposição, em que os efeitos causados pela salinidade foram mais expressivos a partir da concentração $40 \mathrm{~m} \mathrm{~mol} \mathrm{~L}^{-1}$ para ambas as medidas realizadas (Tabela 1).

Tabela 1. Potencial inibitório (PI) e crescimento $(\mathrm{mm})$ da parte aérea e da raiz de plântulas de $P$. vulgaris expostas (144h) a concentrações experimentais de $\mathrm{KCl}$

\begin{tabular}{llll}
\hline $\mathrm{KCl}\left(\mathrm{m} \mathrm{mol} \mathrm{L}^{-1}\right)$ & $\mathrm{PI}$ & Parte aérea & Raiz \\
\hline \multicolumn{1}{c}{ Controle } & - & $15,75 \pm$ & $74,34 \pm 10,42^{\mathrm{a}}$ \\
40 & & $3,62^{\mathrm{a}}$ & \\
80 & $22,67 \pm 4,62^{\mathrm{a}}$ & $8,84 \pm 1,69^{\mathrm{b}}$ & $45,04 \pm 9,49^{\mathrm{b}}$ \\
120 & $45,33 \pm 10,07^{\mathrm{b}}$ & $7,99 \pm 1,21^{\mathrm{b}}$ & $15,58 \pm 3,64^{\mathrm{c}}$ \\
160 & $64,00 \pm 6,93^{\mathrm{c}}$ & $5,45 \pm 1,25^{\mathrm{b}}$ & $7,45 \pm 1,84^{\mathrm{d}}$ \\
200 & $69,33 \pm 6,11^{\mathrm{c}}$ & $2,37 \pm 0,56^{\mathrm{c}}$ & $5,72 \pm 1,41^{\mathrm{de}}$ \\
\hline
\end{tabular}

* Parte aérea não emergiu. Letras minúsculas iguais, para a mesma coluna, indica que os valores médios não apresentaram diferenças estatísticas pelo teste de Tukey $(p<0,05)$.

A biomassa fresca e seca da raiz e da parte aérea das plântulas de $P$. vulgaris foram significativamente a partir de $40 \mathrm{~m} \mathrm{~mol} \mathrm{~L}^{-1}$ do sal, que representou a menor concentração analisada (Figuras 3 e 4). Essa influência na biomassa das partes da plântula foi mais bem explicada pela regressão polinomial, reiterando a correlação entre a biomassa e a concentração salina, por reportar alto coeficientes de determinação ( $R^{2}$ entre 0,93 e 0,99).

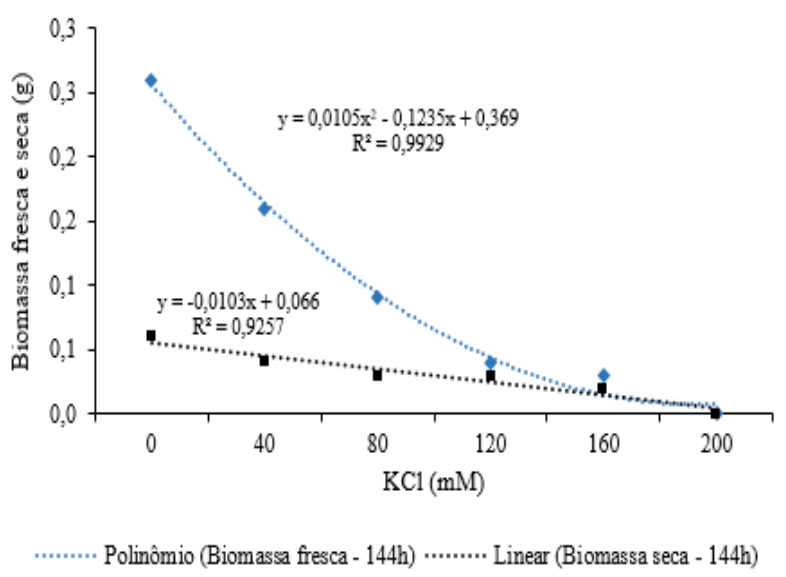

Figura 3. Biomassa fresca e seca da parte aérea de plântulas de $P$. vulgaris, expostas (144h) a concentrações experimentais de $\mathrm{KCl}$

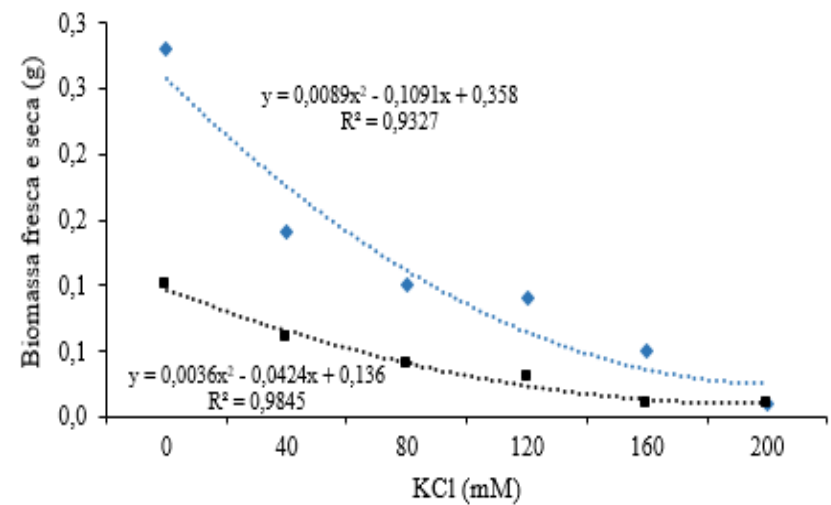

........ Polinômio (Biomassa fresca (144h)) …..... Polinômio (Biomassa seca (144h))

Figura 4. Biomassa fresca e seca da raiz de plântulas de P. vulgaris, expostas (144h) a concentrações experimentais de KCl

\section{DISCUSSÃO}

A presença do $\mathrm{KCl}$ causou efeitos tóxicos ao desenvolvimento germinativo das sementes de $P$. vulgaris, em que tais efeitos são representados, nessa pesquisa, pela redução do IVG, do crescimento, da produção de biomassa e pelo aumento do TMG, fatores que podem ser resultado de diversas alterações bioquímicas e fisiológicas causadas nas sementes desde 0 início da germinação, sendo esses efeitos mais tóxicos nas maiores concentrações analisadas.

0 potássio $(K)$ é um nutriente extremamente importante, se caracterizando como essencial para a sobrevivência dos vegetais, pois participa ativamente de processos fisiológicos diretamente ligados ao crescimento e resistência das plantas (ZÖRB; SENBAYRAM; PEITER, 2014), enquanto que 0 cloro $\left(\mathrm{Cl}^{-}\right)$quando disponível em concentrações elevadas compromete a absorção e uso de inúmeros micronutrientes essenciais ao vegetal (CRUZ et al., 2017)

A redução significativa do crescimento das plântulas de $P$. vulgaris evidenciou que essa espécie sofreu estresse com a exposição à salinidade induzida com KCl, mesmo em pequenas concentrações. Dados similares foram observados na pesquisa de Sá et al. (2017) para a espécie Vigna unguiculata L. (feijãocaupi), em que os autores identificaram que a irrigação 
com água salina reduziu o crescimento em altura das plantas analisadas, reportando a toxidade da salinidade para o desenvolvimento da espécie.

Quando expostas as diferentes concentrações e tipos de sais as espécies podem reagir de formas distintas à variação salina no solo, isso ocorre por que cada espécie tem seu nível de tolerância e mecanismos fisiológicos diferentes (RIBEIR0 et al., 2017). Essas características possibilitam que algumas espécies sobrevivam em um solo com elevadas concentrações salinas, enquanto outras espécies são incapazes de se estabelecer em um solo salino, mesmo que em pequena quantidade.

Ribeiro et al. (2017) concluem ainda que a variação salina das concentrações de sais influencia diretamente na diminuição do potencial hídrico do solo, comprometendo o desenvolvimento da planta, além de estabelecer níveis de toxicidade pelo desequilíbrio no funcionamento metabólico.

Na pesquisa de Souto et al. (2016) foi reportado que 0 teor de salinidade na água utilizada para a irrigação de Morinda citrifolia L. reduziu potencialmente 0 crescimento (altura) da espécie, com efeitos mais danosos a planta na concentração salina de 6,0 dS $\mathrm{m}^{-1}$, em que esse efeito foi atribuído, possivelmente, ao estresse osmótico.

Ribeiro et al. (2017) identificaram que em Erythrina velutina a disponibilidade de cloreto de sódio provocou interferência no funcionamento de atividades bioquímicas e fisiológicas essenciais no processo de germinação e, por isso, o crescimento foi reduzido, causando efeitos mais deletérios ao crescimento da espécie na exposição a concentrações acima de 6 dS.m-1 CE.

A redução da biomassa fresca e seca das plântulas de $P$. vulgaris foi influenciada pela redução do crescimento da raiz e da parte aérea das plântulas que, consequentemente, afetou a produção de biomassa.

A literatura reportou, por meio do trabalho de Sá et al. (2017), que a espécie $V$. unguiculata teve a biomassa seca foliar reduzida quando exposta a água de irrigação com teor de salinidade acima de 1,5 $\mathrm{dSm}^{-1}$.
Dessa forma, o estresse salino quando estabelecido em espécies vegetais pouco adaptadas as características da região semiárida, causam diversos impactos ao desenvolvimento das plântulas, principalmente por estarem associados a outras características físicas da região que não são encontradas em outros ambientes (FREITAS et al., 2014).

Conhecer como as diferentes espécies vegetais reagem a exposição ao estresse salino é importante, pois assim é possível que se possa traçar estratégias mitigadoras de controle de salinidade nas regiões áridas e semiáridas, que são constantemente mais afetadas pelos processos de salinização do solo.

\section{CONCLUSÃO}

0 aumento das concentrações salinas de $\mathrm{KCl}$ interferiu negativamente a germinação, o crescimento e 0 desenvolvimento de $P$. vulgaris, além de reduzir a produção de biomassa mesmo em pequenas concentrações do sal $\left(40 \mathrm{~m} \mathrm{~mol} \mathrm{~L}^{-1}\right)$, evidenciando que essa espécie possui pouca tolerância ao estresse salino causado pelo $\mathrm{KCl}$.

Os solos salinos são, atualmente, uma problemática socioambiental e econômica e, por isso, medidas mitigadoras devem ser desenvolvidas, especialmente nas práticas agrícolas, visando reduzir danos na produtividade de alimentos.

\section{REFERÊNCIAS}

AQUINO, J. P. de A.; BEZERRA, A. A. C. de; ALCÂNTARA NETO, F. de; LIMA, C. J. G. de S.; SOUSA, R. R. de. Morphophysiological responses of cowpea genotypes to irrigation water salinity. Revista Caatinga, Mossoró, v. 30, n. 4, p. 1001-1008, out./dez. 2017.

BEZERRA, I. L.; GHEYI, H. R.; NOBRE, R. G.; LIMA, G. S. de; SANTOS, J. B. dos; FERNANDES, P. D. Interaction between soil salinity and nitrogen on growth and 
gaseous exchanges in guava. Revista Ambiente \& Água, Taubaté, v. 13, n. 3, p. 1-12, 2018.

BRASIL. Ministério da Agricultura. Regra para análises de sementes. Brasília, DF: Ministério da Agricultura, 2009.

CRUZ, J. L.; COELHO FILHO, M. A.; COELHO, E. F.; SANTOS, A. A. dos. Salinity reduces carbon assimilation and the harvest index of cassava plants (Manihot esculenta Crantz). Acta Scientiarum, v. 39, n. 4, p. 545-555, oct./dec. 2017.

DALCHIAVON, F. C.; NEVES, G.; HAGA, K. I. Efeito de stresse salino em sementes de Phaseolus vulgaris. Revista de Ciências Agrárias, v. 39, n. 3, p. 404412, 2016.

FREITAS, M. A. C. et al. Crescimento e tolerância à salinidade em três espécies medicinais do gênero Plectranthus expostas a diferentes níveis de radiação.

Revista Brasileira de Plantas Medicinais, v. 16, n. 4, p. 839-849, 2014.

JOSÉ, A. C.; SILVA, N. C. N.; FARIA, J. M. R.; PEREIRA, W. V. S. Influence of priming on Eucalyptus spp seeds, tolerance to salt stress. Journal of Seed Science, v. 38, n. 4, p. 329-334, oct./dec. 2016.

MAGUIRE, J. Speed of germination aid in selection and evoluation for seedling and vigour. Crop Science, v. 2, p. 176-177, 1962.

OLIVEIRA, W. J.; SOUZA, E. R. de; CUNHA, J. C.; SILVA, E. F. F. e; VELOSO, V. de L. Leaf gas exchange in cowpea and $\mathrm{CO} 2$ efflux in soil irrigated with saline water. Revista Brasileira de Engenharia Agrícola e Ambiental, v. 21, n. 1, p. 32-37, jan. 2017.

PEREIRA, F. A. de L.; MEDEIROS, J. F. de; GHEYI, H. R.; DIAS, N. da S.; PRESTON, W.; VASCONCELOS, C. B. e $\mathrm{L}$. Tolerance of melon cultivars to irrigation water salinity. Revista Brasileira de Engenharia Agrícola e
Ambiental, v. 21, n. 12, p. 846-851, dez. 2017.

RIBEIRO, R. C.; DANTAS, B. F.; MATIAS, J. R.; PELACANI, C. R. Efeito do estresse salino na germinação e crescimento inicial de plântulas de Erythrina velutina Willd. (Fabaceae). Gaia Scientia, v. 11, n. 4, p. 65-78, 2017.

SÁ, F. V. da S.; FERREIRA NETO, M.; LIMA, Y. B. de; PAIVA, E. P. de; GHEYI, H. R.; DIAS, N. da S. Initial development of cowpea plants under salt stress and phosphate fertilization. Ambiente \& Água, v. 12, n. 3, p. 405-415, maio/jun. 2017.

SILVA, M. G. da; OLIVEIRA, I. de S.; SOARES, T. M.; GHEYI, H. R.; SANTANA, G. de 0.; PINHO, J. de $S$. Growth, production and water consumption of coriander in hydroponic system using brackish waters. Revista Brasileira de Engenharia Agrícola e Ambiental, v. 22, n. 8, p. 547-552, ago. 2018.

SOUTO, A. G. de L.; CAVALCANTE, L. F.; LIMA NETO, A. J. de; MESQUITA, F. de 0.; SANTOS, J. B. dos. Biometria em plantas de noni sob irrigação com águas salinas e lixiviação dos sais do solo. Revista Ciência Agronômica, v. 47, n. 2, p. 316-324, abr./jun. 2016.

SOUZA, J. T. A.; NUNES, J. C.; CAVALCANTE, L. F.; NUNES, J. A. da S.; PEREIRA, W. E.; FREIRE, J. L. de 0. Effects of water salinity and organomineral fertilization on leaf composition and production in Passiflora edulis. Revista Brasileira de Engenharia Agrícola e Ambiental, v. 22, n. 8, p. 535-540, ago. 2018.

ZÖRB, C.; SENBAYRAM, M.; PEITER, E. Potassium in agriculture - status and perspectives. Journal of Plant Physiology, v. 171, n. 9, p. 656-669, may 2014

Recebido em: 30/04/2019 Aceito em: 31/05/2019 